\title{
Research on the Intervention Effect of Polydatin of Hyperlipidemia Rats' Early Atherosclerosis
}

\author{
Ouyang Enhong ${ }^{1}$, Junhua Liu², Hailiang Wei ${ }^{1}$, Gengxi Li ${ }^{1}$ \\ ${ }^{1}$ Department of pharmacy, Shaoyang University, Shaoyang Hunan, 422000, China; \\ ${ }^{2}$ Department of Pharmacology, Affiliated Hospital of Shaoyang University, Shaoyang Hunan, 422000, \\ China
}

Keywords: Polydatin, Atherosclerosis, Hyperlipidemia.

\begin{abstract}
Purpose: to discuss the function of polydatin on regulating blood-lipid and anti-atherosclerosis. Method: feed rats with high-fat diet to establish early atherosclerosis model, after the model is established successfully, feed with high, medium and low dose of polydatin (160, $\left.80,40 \mathrm{mg} \cdot \mathrm{kg}^{-1} \cdot \mathrm{d}^{-1}\right), 8$ weeks later, measure the rats' serum cholesterol (TC), triglyceride (TG), HDL-Cholesterol (HDL-C), LDL-Cholesterol (LDL-C), tumor necrosis factor (TNF- $\alpha$ ), nitric oxide (NO) and inducible nitric oxide synthase (iNOS); take thoracic aorta to carry out oil red $\mathrm{O}$ dying to observe the deposition of aortic intimal lipid; take live to make regular HE dying to observe hepatic pathological changes. Result: high, medium and low dosage of polydatin can obviously reduce hyperlipidemia rats' serum TC, TG, LDL-C, TNF- $\alpha$, NO level and iNOS activity, improve HDL-C level, the difference is statistically significant $(\mathrm{P}<0.05)$. Meanwhile, polydatin can decrease the intraaortic leather fat deposition, improve the fatty degeneration and inflammatory cell infiltration of liver. Conclusion: polydatin has functions on regulating blood fat, decreasing the happen of atherosclerosis. This function may relate with its adjust NO and the formation of synthase.
\end{abstract}

\section{Introduction}

This experiment observes the function of polidatin on regulation blood fat and atherosclerosis through establishing rat atherosclerosis model.

\section{Materials}

\section{Drugs and reagents}

Polydatin ( Batch No.: 150731, provided by Shanghai Pure One Biotechnology Co., Ltd. Content >98\%); simvastatin (Batch No.: 150292, Shanghai MSD Pharmaceutical Co., Ltd. ); TC reagent (Batch No.: 20150907), TG reagent (Batch No.: 20150728), HDL-C reagent (Batch No.:20150910) , LDL-C reagent (Batch No.: 20150729), provided by sigma company; TNF- $a$ ELISA reagent (American R\&D Company); NO reagent (Batch No.: 20151117, Nanjing Jiancheng Bioengineering Research Institution); iNOS reagent (Batch No.: 20150112, Nanjing Jiancheng Bioengineering Research Institution).

\section{Instrument}

BIO-RAD Model680 microplate reader (Bio-Rad Co., Ltd); Mikro22R high speed freezing centrifuge (Germany Hettich company).

\section{Animal}

Male SD rats, with weight of 200-250g, provided by Jinan Jinfeng Experimental Animal Breeding Co., Ltd, license No.: SCXK(lu)20150006. 


\section{Diet}

High fat feed formula: $3 \%$ cholesterol, $0.5 \%$ sodium cholate, $10 \%$ lard oil, $0.2 \%$ propylthiouracil, $5 \%$ white sugar, $81.3 \%$ basal feed.

\section{Method}

Establishment and grouping of hyperlipidemia rats model.

SD rats are randomly divided into 6 groups, each group 10 rats. Feed the control group with normal feed, other groups with high fat feed. After 4 weeks, take blood sample from eye socket to measure blood fat, then randomly divide high fat diet groups into 5 groups according to the blood fat level: (1) model group, ig distilled water. (2) Positive drug simvastatin group: ig $5 \mathrm{mg} \bullet \mathrm{kg}-1 \cdot \mathrm{d}-1$. (3) High dosage polydation group: ig $160 \mathrm{mg} \cdot \mathrm{kg}^{-1} \cdot \mathrm{d}^{-1}$. (4)Medium dosage polydation group: ig 80 $\mathrm{mg} \cdot \mathrm{kg}^{-1} \cdot \mathrm{d}^{-1}$. (5)Low dosage polydation group: ig $40 \mathrm{mg} \cdot \mathrm{kg}^{-1} \cdot \mathrm{d}^{-1}$. Feed drugs (or distilled water ) according to groups since the $5^{\text {th }}$ week, feed for 8 weeks, feed normal control group with normal feed, other groups with high fat feed.

\section{Weight measure}

Record weight in the $4^{\text {th }}, 8^{\text {th }}, 12^{\text {th }}$ week, respectively before and after the model establishment.

\section{Take blood sample from the orbital venous}

After the last drug feeding, place for 30 min then centrifuge to separate serum, test relevant indicators according to the instruction book of the reagents.

\section{Observation of histomorphology}

Kill the experimental animal after feeding the final drug. Take liver and fix with 4\% paraformaldehyde solution, embed and slice with routine paraffin, HE dying, observe under the light microscope. Take thoracic aorta slice up with freezing microtome for $10 \mu \mathrm{m}$, immerse into the oil red O solution for $5 \mathrm{~min}$, use $60 \%$ isopropanol to wash up excess dye solution, wash with distilled water, use hematoxylin to dye karyon slightly, use 1\% hydrochloric acid water to slightly differentiate, wash with running water for $10 \mathrm{~min}$, seal sheet with glycerin gelatine and observe under the light microscope.

\section{Data processing}

All the experimental materials are expressed with $\bar{x} \pm \mathrm{s}$, use ANOVA variance analysis among groups, when $\mathrm{P}<0.05$, it has significant difference.

\section{Result}

\section{Weight change of rats in different groups}

Weight of rats in the normal control group increased gradually, that of the model group increased slowly, obviously lighter than the normal control group $(\mathrm{P}<0.01)$, weight of the simvastatin group and high dosage of polydatin group was increased a little than the model group, but has no significant difference. See Table 1 for the results. 
Table 1 . Weight change of rats in the 4th , 8th , 12th week before and after modeling

$$
(\mathrm{n}=10, \bar{x} \pm \mathrm{s})
$$

\begin{tabular}{|c|c|c|c|c|c|}
\hline \multirow{2}{*}{ Groups } & \multirow{2}{*}{$\begin{array}{c}\text { Dosage } \\
(\mathrm{mg} \cdot \mathrm{kg}-1)\end{array}$} & \multicolumn{4}{|c|}{ Weight/g } \\
\hline & & Before fed with drugs & The 4th week & The 8th week & The 12th week \\
\hline Normal control group & & $212.5 \pm 13.0$ & $290.6 \pm 25.3$ & $314.3 \pm 18.0$ & $386.2 \pm 16.5$ \\
\hline Model group & & $201.8 \pm 11.0$ & $\begin{array}{c}217.9 \pm 16.6 \\
*\end{array}$ & $\begin{array}{c}225.4 \pm 15.0 \\
*\end{array}$ & $230.2 \pm 10.4 *$ \\
\hline Simvastatin group & 5 & $199.7 \pm 8.9$ & $217.2 \pm 18.4$ & $229.6 \pm 14.6$ & $\begin{array}{c}241.8 \pm 20.6 * \\
*\end{array}$ \\
\hline High dosage of polydatin group & 160 & $206.7 \pm 13.0$ & $220,3 \pm 15.6$ & $228.0 \pm 19.0$ & $241.9 \pm 20.8$ \\
\hline Medium dosage of polydatin group & 80 & $210.0 \pm 11.6$ & $216.2 \pm 15.7$ & $224.0 \pm 7.8$ & $228.2 \pm 15.7$ \\
\hline Low dosage of polydatin group & 40 & $204.6 \pm 11.8$ & $225.6 \pm 16.2$ & $228.1 \pm 8.9$ & $224.5 \pm 15.9$ \\
\hline
\end{tabular}

* Compared with the normal control group: $\mathrm{P}<0.01$; * Compared with the model control group: $\mathrm{P}<0.05$

\section{Influence of polydatin on rats' blood fat}

Compared with normal control group, the serum TC, TG, LDL-C content of rats in the model group is obviously increased ( $\mathrm{P}<0.01$ ), HDL-C content is obviously decreased, HDL-C is obviously improved, and presents dosage correlation. See Table 2 for the results.

Table 2. The influence of polydatin on Rats' serum TC, TG, LDL, HDL ( $n=10, \pm s)$

\begin{tabular}{|c|c|c|c|c|c|}
\hline Groups & Dosage $(\mathrm{mg} \cdot \mathrm{kg}-1)$ & $\begin{array}{c}\mathrm{TG}(\mathrm{mmol} \cdot \mathrm{L}-1 \\
)\end{array}$ & $\mathrm{TC}(\mathrm{mmol} \cdot \mathrm{L}-1)$ & $\mathrm{LDL}(\mathrm{mmol} \cdot \mathrm{L}-1)$ & $\begin{array}{c}\mathrm{HDL}(\mathrm{mmol} \cdot \mathrm{L}-1 \\
)\end{array}$ \\
\hline Normal control group & & $0.542 \pm 0.100 * *$ & $\begin{array}{c}1.542 \pm 0.100 * \\
*\end{array}$ & $0.554 \pm 0.091 * *$ & $1.403 \pm 0.144^{* *}$ \\
\hline Model group & & $1.940 \pm 0.086$ & $2.782 \pm 0.267$ & $1.432 \pm 0.113$ & $0.886 \pm 0.056$ \\
\hline Simvastatin group & 5 & $1.814 \pm 0.076^{* *}$ & $2.492 \pm 0.321 *$ & $0.803 \pm 0.116^{* *}$ & $1.329 \pm 0.167 * *$ \\
\hline High dosage of polydatin group & 160 & $1.753 \pm 0.078 * *$ & $\begin{array}{c}2.256 \pm 0.099 * \\
*\end{array}$ & $0.874 \pm 0.046^{* *}$ & $1.298 \pm 0.110 * *$ \\
\hline $\begin{array}{l}\text { Medium dosage of polydatin } \\
\text { group }\end{array}$ & 80 & $1.814 \pm 0.089 * *$ & $2.524 \pm 0.104^{*}$ & $1.084 \pm 0.040 * *$ & $1.004 \pm 0.111^{*}$ \\
\hline Low dosage of polydatin group & 40 & $1.831 \pm 0.089 *$ & $2.608 \pm 0.118$ & $1.298 \pm 0.108 *$ & $0.968 \pm 0.114$ \\
\hline
\end{tabular}

Note: compared with the model group, ${ }^{*} \mathrm{P}<0.05, * * \mathrm{P}<0.01$

\section{Influence of polydatin on Rats' serum TNF- $\alpha$, NO and iNOS}

Compared with normal control group, the serum TNF- a level of rats in the model group is obviously increased ( $\mathrm{P}<0.01$ ). Compared with the model control group, high dosage of polydatin group and medium dosage of polydatin group can obviously decrease TNF- $a$ level $(P<0.05)$ and presents dosage correlation. Compared with normal groups, serum NO, iNOS of rats in model group is obviously improved $(\mathrm{P}<0.01)$. Compared with the model group, the NO content and iNOS activity in the high and medium dosage of polydatin group are obviously decreased, with significant difference $(\mathrm{P}<0.05)$. See Table 3 for the results. 
Table 3. Influence of polydatin on Rats' $\quad$ serum TNF- $\alpha$, NO and iNOS express ( $\left.n=10, \bar{x}_{ \pm s}\right)$

\begin{tabular}{|c|c|c|c|c|}
\hline Groups & $\begin{array}{c}\text { Dosage } \\
(\mathrm{mg} \cdot \mathrm{kg}-1)\end{array}$ & $\begin{array}{l}\text { TNF- a } \\
(\mathrm{ng} \cdot \mathrm{L}-1)\end{array}$ & $\begin{array}{c}\mathrm{NO} \\
(\mathrm{mmol} \cdot \mathrm{mL}-1) \\
\end{array}$ & $\begin{array}{c}\text { iNOS } \\
(\mathrm{U} \cdot \mathrm{mL}-1) \\
\end{array}$ \\
\hline Normal control group & & $\begin{array}{c}60.230 \pm 1.254 * \\
*\end{array}$ & $\begin{array}{c}4.320 \pm 0.471^{*} \\
*\end{array}$ & $\begin{array}{c}8.680 \pm 0.514 * \\
*\end{array}$ \\
\hline Model group & & $95.620 \pm 4.216$ & $11.400 \pm 0.775$ & $13.400 \pm 3.582$ \\
\hline Simvastatin group & 5 & $\begin{array}{c}76.660 \pm 4.021 * \\
*\end{array}$ & $\begin{array}{c}5.070 \pm 0.554 * \\
*\end{array}$ & $\begin{array}{c}8.200 \pm 1.770 * \\
*\end{array}$ \\
\hline High dosage of polydatin group & 160 & $\begin{array}{c}82.820 \pm 4.578 * \\
*\end{array}$ & $\begin{array}{c}6.830 \pm 1.287 * \\
*\end{array}$ & $\begin{array}{c}8.310 \pm 0.649 * \\
*\end{array}$ \\
\hline Medium dosage of polydatin group & 80 & $91.090 \pm 3.813^{*}$ & $\begin{array}{c}9.420 \pm 0.928 * \\
*\end{array}$ & $9.850 \pm 1.613^{*}$ \\
\hline Low dosage of polydatin group & 40 & $93.578 \pm 4.067$ & $\begin{array}{c}10.130 \pm 1.177 \\
*\end{array}$ & $11.140 \pm 1.386$ \\
\hline
\end{tabular}

Note: compared with the model group, ${ }^{*} \mathrm{P}<0.05$, $* * \mathrm{P}<0.01$

\section{Influence of polydatin on rats' liver morphology}

Through visual inspection, the liver color of rats in the normal group is evenly dark red, the texture is smooth. That of the model group is yellowish-brown, the texture is fat. The liver morphological change of the Simvastatin group and polydatin groups is slight. Observe the HE dying liver tissue sections, the hepatic lobule structure of the normal group is normal, hepatic cords are in alignment and are radiated, the nucleus structure is clear, no hepatic cell fattydegeneration and inflammatory cell infiltration. Liver of rats in the model group showed fatty degeneration, the hepatic lobule structure was damaged, hepatic cords were in disorder, liver cells are markedly swollen, there are lot of fat accumulation in the cell, Nucleus was squeezed in cell edge. The hepatocyte adipose degeneration in the Simvastatin group and polydatin group is obviously relieved, see lipid droplet and vacuole incidentally.

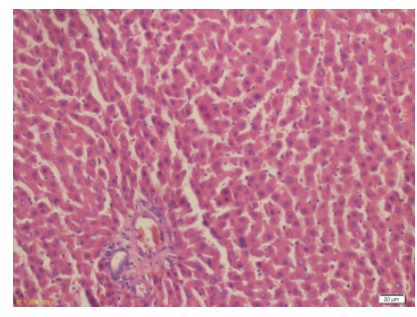

Blank control group

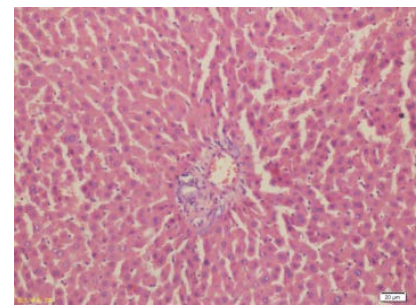

High dosage of polydatin group

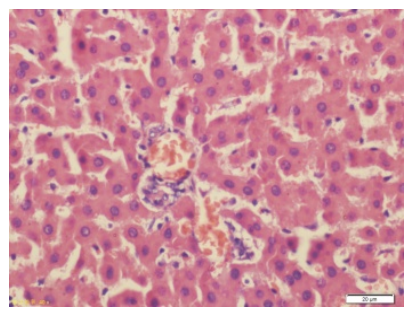

Model group

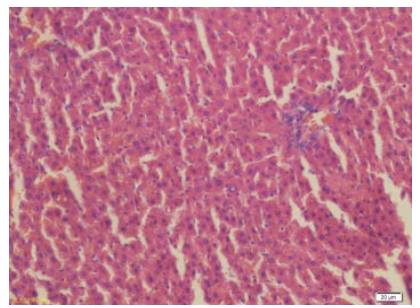

Medium dosage of polydatin group

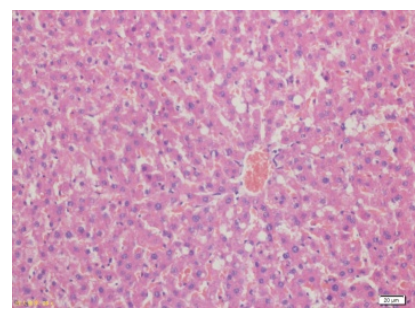

Simvastatin group

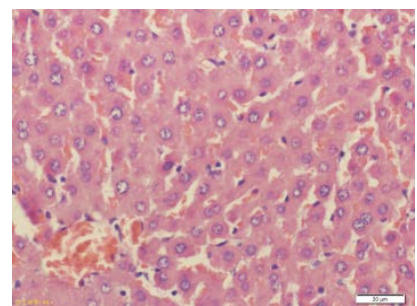

Low dosage of polydatin group

Fig. 1. Observation on the Liver histopathology of rats in different groups (HE dying $\times 200$, arrows indicate fat cavitation)

\section{Influence of polydatin on the atherosclerotic lesion}

The endarterium of rats in the normal group is smooth, no oil red $\mathrm{O}$ dying lipid deposition area. The arterial wall of the model group has lipidosis, mainly under the intima. Simvastatin group and 
polydatin groups can reduce arterial wall lipid deposition, among which, after oil red dying, the arterial wall of Simvastatin group , high dosage of polydatin group and normal group almost has no difference.

\section{Discussion}

Numerous studies have demonstrated that high levels of TC, TG, LDL-C can stimulate the formation and development of AS, while high level of HDL-C can reduce this risk ${ }^{[1]}$. In this experiment, the high dosage of polydatin group and medium dosage of polydatin group can obviously reduce serum TC, TG, LDL-C level, improve HDL-C, has obvious improvement and regulating effect on lipid metabolism disorder. The pathological histology observation shows that polydatin can relieve the cholesterol accumulation of the periphery tissues, prevent the formation of AS nidus, to protect the liver from fat deposition, which has protection on the liver diffuse fatty change and AS lesion of the high-fat animals. "injury responsive theory" thought that chronic inflammatory process is one of the main features of AS, they interact with each other through immunoreaction ${ }^{[2]}$. TNF- $a$ is the clear inflammatory indicators that take parts in AS formation, evolution and fracture ${ }^{[3]}$. High and medium dosage of polydatin can obviously reduce serum TNF- a level, polydatin has the function of anti-AS through prohibiting the inflammatory response in AS development. iNOS is the key enzyme $^{[4]}$ produced by NO in the body, NO/iNOS has close relation with atherosclerosis and other cardiovascular disease $^{[5]}$. The NO content and iNOS activity of the polydatin group has some reduce, which shows that it has anti-inflammatory action.Experimental results show that polydatin can give play to its cure for the AS of rats through balancing the blood lipid metabolism, intervening inflammatory response and regulating the generation of NO.

\section{Acknowledgments}

This paper is the funding project of Hunan Provincial Education Department, project No.: 13 C871.

\section{References}

[1] PETER P. High-density lipoprotein as a therapeutic target: clinicalevidence and treatment strategies. Am J Cardiol,2005,96( 9) : 50 -58.

[2] Wang Xiaomei, Si liangyi. The Influence of Endotoxi on the Rats' Atherosclerotic Lesions and the Function of TLR4. Third Military Medical University Journals, 2006,28 (23) :2368 -2370.

[3] Cheng Wenli. Recent Progress of Research on the Stability of Serum Inflammation Markers and Artery Atherosclerotic Llaque. Advances in Cardiovascular Diseases, 2005,26( 3) : 262 -265.

[4] Ulrich F,William CS. Nitric oxide synthases: regulation and function. Eur Hart J, 2012,33(7) : 829-837.

[5] RUBBO H,O'DONNELL V. Nitric oxide,peroxynitrite and lipoxygenasein atherogenesis: mechanistic insights. Toxicology,2005,208(2) : 305-317. 